УДК $517.98,517.5$

\author{
N. S. SushchyK, V. M. Degnerys
}

\title{
FACTORISATION OF ORTHOGONAL PROJECTORS
}

\begin{abstract}
N. S. Sushchyk, V. M. Degnerys. Factorisation of orthogonal projectors, Mat. Stud. 55 (2021), 181-187.

We study the problem of a special factorisation of an orthogonal projector $P$ acting in the Hilbert space $L_{2}(\mathbb{R})$ with $\operatorname{dim} \operatorname{ker} P<\infty$. In particular, we prove that the orthogonal projector $P$ admits a special factorisation in the form $P=V V^{*}$, where $V$ is an isometric upper-triangular operator in the Banach algebra of all linear continuous operators in $L_{2}(\mathbb{R})$. Moreover, we give an explicit formula for the operator $V$.
\end{abstract}

1. Introduction. Let $H:=L_{2}(\mathbb{R})$ be a Hilbert space with the standard norm $\|\cdot\|$ and the inner product $(\cdot \mid \cdot)$, and let $\mathcal{B}:=\mathcal{B}(H)$ be the Banach algebra of all linear continuous operators in $H$. Let us fix in the algebra $\mathcal{B}$ the continuous chain of orthoprojectors $\mathfrak{E}:=$ $\{E(\xi)\}_{\xi \in \mathbb{R}}$, where $E(\xi)$ is the multiplication operator on the characteristic function of the interval $(-\infty, \xi)$.

An operator $A \in \mathcal{B}$ is called an upper-triangular operator with respect to the chain $\mathfrak{E}$ if for every $E \in \mathfrak{E}$ the subspace $E H$ is an invariant subspace of $A$, i,e.,

$$
E^{\perp} A E=0, \quad E \in \mathfrak{E} \quad\left(E^{\perp}:=I-E\right) .
$$

Similarly, an operator $A \in \mathcal{B}$ is called a lower-triangular operator with respect to the chain $\mathfrak{E}$ if for every $E \in \mathfrak{E}$ the subspace $E^{\perp} H$ is an invariant subspace of $A$, i.e.,

$$
E A E^{\perp}=0, \quad E \in \mathfrak{E} .
$$

We set

$$
\begin{array}{ll}
\mathcal{B}^{+}:=\{B \in \mathcal{B}: \forall E \in \mathfrak{E} & \left.E^{\perp} B E=0\right\} \\
\mathcal{B}^{-}:=\{B \in \mathcal{B}: \forall E \in \mathfrak{E} & \left.E B E^{\perp}=0\right\}
\end{array}
$$

$\mathcal{B}^{+}$and $\mathcal{B}^{-}$are closed subalgebras in the algebra $\mathcal{B}$. It is easy to see that if $A \in \mathcal{B}^{+}$, then the adjoint operator $A^{*}$ belongs to the algebra $\mathcal{B}^{-}$.

Definition 1. We say that an operator $A \in \mathcal{B}$ admits $U L$-factorisation if there exist $A_{+} \in$ $\mathcal{B}^{+}, A_{-} \in \mathcal{B}^{-}$such that $A=A_{+} A_{-}$.

2010 Mathematics Subject Classification:47A68, 47A46.

Keywords: special factorisation; orthogonal projector.

doi:10.30970/ms.55.2.181-187

(C) N. S. Sushchyk, V. M. Degnerys, 2021 
Definition 1 is wider than usually accepted in the mathematical literature since it does not assume invertibility of $A$ (see, e.g., [1]). We only know the paper [2], where the factorisation problem was studied for nonnegative non-invertible operators.

In the case when $A \in \mathcal{B}$ is a nonnegative self-adjoint operator, we consider a special factorisation.

Definition 2. Let $A \in \mathcal{B}$ and $A \geq 0$. We say that $A$ admits a special factorisation if there exists $A_{+} \in \mathcal{B}^{+}$such that $A=A_{+} A_{+}^{*}$.

In the present paper, we study the following problem.

Problem 1. Does every orthogonal projector $P \in \mathcal{B}$ with $\operatorname{dim}$ ker $P<\infty$ admit a special factorisation in the form $P=V V^{*}$, where $V$ is an isometric operator in $\mathcal{B}^{+}$?

It follows from the results of Larson [3] that not every uniformly positive operator $A \in \mathcal{B}$ admits a factorisation $A=B B^{*}$, where $B \in \mathcal{B}^{+}$. In the case when an operator is noninvertible the problem of its special factorisation is much more difficult. In the mentioned work [2], a special factorisation of an orthogonal projector $P$ with $\operatorname{dim} \operatorname{ker} P<\infty$ was considered in the Hilbert space $L_{2}(0,1)$ with chain of orthoprojectors $\{\widetilde{E}(\xi) \mid \xi \in[0,1]\}$, where $\widetilde{E}(\xi)$ is the multiplication operator on the characteristic function of the interval $[0, \xi)$. In [2], it was proved that an orthogonal projector $P$ with $\operatorname{dim} \operatorname{ker} P<\infty$ admits a special factorisation if the additional condition

$$
\forall \xi \in[0,1] \quad \operatorname{dim} \widetilde{E}(\xi) \operatorname{ker} P=\operatorname{dim} \operatorname{ker} P
$$

holds.

The main result of this paper gives an explicit formula for an isometric operator $V \in \mathcal{B}^{+}$ such that $V V^{*}=P$ for an orthogonal projector $P \in \mathcal{B}$ with $\operatorname{dim} \operatorname{ker} P<\infty$.

2. Factorisation of an orthogonal projector. Denote by $G$ the Hilbert space $\mathbb{C}^{n}$ with the standard inner product

$$
(x \mid y)_{G}:=\sum_{j=1}^{n} x_{j} \bar{y}_{j}, \quad x=\left(x_{j}\right)_{j=1}^{n}, y=\left(y_{j}\right)_{j=1}^{n} .
$$

Let $P$ be an orthogonal projector in $\mathcal{B}$ with $\operatorname{dim} \operatorname{ker} P=n \in \mathbb{N}$, and let $\left(\varphi_{j}\right)_{j=1}^{n}$ be an orthonormal basis in the space ker $P$.

Let us consider the function $\Phi: \mathbb{R} \rightarrow G^{\prime}$ ( $G^{\prime}$ is the dual space to $G$ ) that is defined by the formula

$$
\Phi(t) c=\sum_{j=1}^{n} c_{j} \varphi_{j}(t), \quad t \in \mathbb{R}, \quad c=\left(c_{j}\right)_{j=1}^{n} \in G .
$$

It is easy to see that $\Phi \in L_{2}\left(\mathbb{R}, G^{\prime}\right)$. Denote by $\Phi^{*}(t)$ the operator that is adjoint to $\Phi(t)$. It acts from $\mathbb{C}$ into $G$ by the formula

$$
\Phi^{*}(t) c=c\left(\overline{\varphi_{1}(t)}, \ldots, \overline{\varphi_{n}(t)}\right), \quad t \in \mathbb{R}, \quad c \in \mathbb{C} .
$$

We also define the function

$$
A(x):=\int_{-\infty}^{x} \Phi^{*}(t) \Phi(t) d t, \quad x \in \mathbb{R}
$$


which plays an important role in the study. Clearly, this function is absolutely continuous, and $A^{\prime}(x)=\Phi^{*}(x) \Phi(x)$ for almost every $x \in \mathbb{R}$. Moreover, $A^{*}(x)=A(x)$ for all $x \in \mathbb{R}$, and

$$
A\left(x_{1}\right) \leq A\left(x_{2}\right) \quad \text { for } \quad x_{1} \leq x_{2}
$$

Let us consider the subspaces

$$
F(x):=\operatorname{ker} A(x), \quad G(x):=\operatorname{ran} A(x), \quad x \in \mathbb{R} .
$$

Since $G(x) \oplus F(x)=G$ for all $x \in \mathbb{R}$, it follows by (2) that

$$
F\left(x_{1}\right) \supset F\left(x_{2}\right), \quad G\left(x_{1}\right) \subset G\left(x_{2}\right) \quad \text { for } \quad x_{1} \leq x_{2} .
$$

It is easy to check that the function

$$
\rho(x):=\operatorname{dim} F(x), \quad x \in \mathbb{R},
$$

is nonincreasing, left-continuous, and piecewise-constant. We denote by $\left(\xi_{k}\right)_{k=1}^{m}$ a strictly increasing sequence of all points of discontinuity of the function $\rho$, and let

$$
\Delta_{s}:= \begin{cases}\left(-\infty, \xi_{1}\right), & \text { if } s=1 \\ \left(\xi_{s-1}, \xi_{s}\right), & \text { if } 1<s \leq m \\ \left(\xi_{m},+\infty\right), & \text { if } s=m+1\end{cases}
$$

Put $F_{k}:=F\left(\xi_{k}\right), G_{k}:=G\left(\xi_{k}\right), k=1, \ldots, m$. It follows from the above that

$$
F(x)=F_{k}, \quad G(x)=G_{k}, \quad x \in \Delta_{k}, \quad k=1, \ldots, m,
$$

and, moreover, $F(x)=\{0\}=: F_{m+1}, G(x)=G=: G_{m+1}, \quad x \in \Delta_{m+1}$. Thus the equalities

$$
\operatorname{ran} A(x)=G_{k}, \quad x \in \Delta_{k}, \quad k=1, \ldots, m+1,
$$

hold.

Lemma 1. For an arbitrary $x \in \Delta_{k}(k=1, \ldots, m+1)$ the operator

$$
A_{k}(x):=\left.A(x)\right|_{G_{k}}
$$

is invertible in $G_{k}$. Moreover, the function $x \mapsto A_{k}^{-1}(x)$ is continuous on $\Delta_{k}$.

Proof. Let $x \in \Delta_{k}$. Since the operator $A(x)$ is self-adjoint, it follows from (3) that $A$ maps $G_{k}$ onto itself. Hence the operator $A_{k}(x)$ is invertible. As already mentioned, the function $x \rightarrow A(x)$ is continuous. Thus the function $\Delta_{k} \ni x \rightarrow A_{k}(x)$ is continuous, too. Taking into account that the operators $A_{k}(x), x \in \Delta_{k}$, are invertible, we obtain that the functions $x \mapsto A_{k}^{-1}(x)$ are continuous on $\Delta_{k}$.

Definition 3. Denote by $A^{b}: \mathbb{R} \rightarrow \mathcal{B}(G)$ the function acting by the formula

$$
A^{b}(x):=A_{k}^{-1}(x) \oplus O_{k}, \quad x \in \Delta_{k}, \quad k=1, \ldots, m+1,
$$

where $O_{k}$ is a null-operator in $F_{k}$. 
Remark 1. It follows from Lemma 1 that the function $x \mapsto A^{b}(x)$ is continuous on every interval $\Delta_{j}$, and its points of discontinuity can only be points $\xi_{j}, j=1, \ldots, m$. Moreover, in view of Lemma 1 and (1), we have for almost every $x \in \Delta_{k}(k=1, \ldots, m+1)$ the equality

$$
A(x) A^{b}(x) \Phi^{*}(x)=\Phi^{*}(x) .
$$

The main result of this paper is:

Theorem 1. The formula

$$
(V f)(x):=f(x)-\int_{x}^{\infty} \Phi(x) A^{b}(t) \Phi^{*}(t) f(t) d t, \quad x \in \mathbb{R}, \quad f \in H,
$$

defines an isometric upper-triangular operator such that $V V^{*}=P$.

The proof of Theorem 1 will be divided into parts presented below as lemmas. Denote by $C_{0}$ the set of all continuous functions $f: \mathbb{R} \rightarrow \mathbb{C}$ with compact support not intersecting the set $\left\{\xi_{j}\right\}_{j=1}^{m}$. Note that the set $C_{0}$ is everywhere dense in $H$.

Lemma 2. Assume that the operator $V$ is introduced by the formula (5) and $U=I-V$. For an arbitrary $f \in C_{0}$ the equality

$$
\|U f\|^{2}=(U f \mid f)+(f \mid U f)
$$

holds.

Proof. Let $f \in C_{0}$. Taking into account Remark 1, we conclude that the vector-valued function

$$
h(t):=A^{b}(t) \Phi^{*}(t) f(t), \quad t \in \mathbb{R},
$$

is square integrable and has a compact support; as a result, it is integrable on $\mathbb{R}$. Hence the function

$$
x \rightarrow \int_{x}^{\infty} h(t) d t
$$

is continuous and bounded on the whole line. Thus, since the function $x \mapsto\|\Phi(x)\|$ belongs to $H$, we obtain that $U f \in H$ and

$$
(U f)(x)=\int_{x}^{\infty} \Phi(x) h(t) d t, \quad x \in \mathbb{R} .
$$

It follows from the last formula that

$$
|(U f)(x)|^{2}=\int_{x}^{\infty} \int_{x}^{\infty}\left(\Phi^{*}(x) \Phi(x) h(t) \mid h(\xi)\right)_{G} d t d \xi .
$$

Let us calculate the integral

$$
J:=\int_{\mathbb{R}}|(U f)(x)|^{2} d x=\int_{-\infty}^{\infty} \int_{x}^{\infty} \int_{x}^{\infty}\left(\Phi^{*}(x) \Phi(x) h(t) \mid h(\xi)\right)_{G} d t d \xi d x .
$$

We see that $J=J_{1}+J_{2}$, where

$$
\begin{aligned}
& J_{1}:=\iiint_{x \leq t \leq \xi}\left(\Phi^{*}(x) \Phi(x) h(t) \mid h(\xi)\right)_{G} d x d t d \xi, \\
& J_{2}:=\iiint_{x \leq \xi \leq t}\left(\Phi^{*}(x) \Phi(x) h(t) \mid h(\xi)\right)_{G} d x d t d \xi .
\end{aligned}
$$


Integrating over the variable $x$ the integrals $J_{1}$ and $J_{2}$, and taking into account the definition of the operator $A(x)$, we get that

$$
\begin{gathered}
J_{1}=\iint_{t \leq \xi}(A(t) h(t) \mid h(\xi))_{G} d t d \xi \\
J_{2}=\iint_{\xi \leq t}(A(\xi) h(t) \mid h(\xi))_{G} d t d \xi=\iint_{\xi \leq t}(h(t) \mid A(\xi) h(\xi))_{G} d t d \xi .
\end{gathered}
$$

Note that, for almost every $t \in \mathbb{R}$,

$$
A(t) h(t)=A(t) A^{b}(t) \Phi^{*}(t) f(t)=\Phi^{*}(t) f(t) .
$$

Thus

$$
J_{1}=\iint_{t \leq \xi}\left(\Phi^{*}(t) f(t) \mid h(\xi)\right)_{G} d t d \xi=\iint_{t \leq \xi}(f(t) \mid \Phi(t) h(\xi))_{G} d t d \xi=(f \mid U f) .
$$

Similarly, we obtain that

$$
J_{2}=\iint_{\xi \leq t}\left(h(t) \mid \Phi^{*}(\xi) f(\xi)\right)_{G} d t d \xi=\iint_{\xi \leq t}(\Phi(\xi) h(t) \mid f(\xi))_{G} d t d \xi=(U f \mid f) .
$$

Therefore, $J=(U f \mid f)+(f \mid U f)$ as claimed.

Corollary 1. The operator $V$ that is defined by the formula (5) is an isometric uppertriangular operator.

Proof. According to Lemma 2, we get that for an arbitrary $f \in C_{0}$

$$
\|V f\|^{2}=(f-U f \mid f-U f)=\|f\|^{2}+\|U f\|^{2}-(U f \mid f)-(f \mid U f)=\|f\|^{2} .
$$

Since the set $C_{0}$ is everywhere dense in $H$, we have that the operator $V$ is continuously extended to an isometric operator on the whole space $H$. Obviously, the extended operator acts by the formula (5) and it is upper-triangular.

Lemma 3. For every $g \in C_{0}$ the equality

$$
\left\|U^{*} g\right\|^{2}=\left(U^{*} g \mid g\right)+\left(g \mid U^{*} g\right)-\left\|P^{\perp} g\right\|^{2} \quad\left(P^{\perp}:=I-P\right)
$$

holds.

Proof. Using elementary calculations, we get that the adjoint to $U$ operator acts on functions $g \in C_{0}$ by the formula

$$
\left(U^{*} g\right)(x)=\int_{-\infty}^{x} \Phi(x) A^{b}(x) \Phi^{*}(t) g(t) d t, \quad x \in \mathbb{R} .
$$

Thus for an arbitrary $g \in C_{0}$

$$
\left|\left(U^{*} g\right)(x)\right|^{2}=\left|\int_{-\infty}^{x} \Phi(x) A^{b}(x) \Phi^{*}(t) g(t) d t\right|^{2}=\left(A^{b}(x) \Phi^{*}(x) \Phi(x) A^{b}(x) r(x) \mid r(x)\right)_{G},
$$


where

$$
r(x):=\int_{-\infty}^{x} \Phi^{*}(t) g(t) d t, \quad x \in \mathbb{R}
$$

Taking into account (4), it is easy to check that for almost every $x \in \mathbb{R}$

$$
\left(A^{b}(x)\right)^{\prime}=-A^{b}(x) A^{\prime}(x) A^{b}(x)=-A^{b}(x) \Phi^{*}(x) \Phi(x) A^{b}(x) .
$$

Thus

$$
\left|\left(U^{*} g\right)(x)\right|^{2}=-\left(\left(A^{b}(x)\right)^{\prime} r(x) \mid r(x)\right)_{G}=-\int_{-\infty}^{x} \int_{-\infty}^{x}\left(\left(A^{b}(x)\right)^{\prime} \Phi^{*}(t) g(t) \mid \Phi^{*}(\xi) g(\xi)\right)_{G} d t d \xi .
$$

Using this fact, we get

$$
\left\|U^{*} g\right\|^{2}=J:=-\int_{-\infty}^{\infty} \int_{-\infty}^{x} \int_{-\infty}^{x}\left(\left(A^{b}(x)\right)^{\prime} \Phi^{*}(t) g(t) \mid \Phi^{*}(\xi) g(\xi)\right)_{G} d t d \xi d x .
$$

Similarly to the proof of Lemma 2, we rewrite the integral $J$ as a sum of the integrals

$$
\begin{aligned}
& \left.J_{1}:=-\iiint_{\xi \leq t \leq x}\left(A^{b}(x)\right)^{\prime} \Phi^{*}(t) g(t) \mid \Phi^{*}(\xi) g(\xi)\right)_{G} d t d \xi d x, \\
& \left.J_{2}:=-\iiint_{t \leq \xi \leq x}\left(A^{b}(x)\right)^{\prime} \Phi^{*}(t) g(t) \mid \Phi^{*}(\xi) g(\xi)\right)_{G} d t d \xi d x .
\end{aligned}
$$

Integrating over the variable $x$ in both integrals and using that

$$
\int_{t}^{+\infty}\left(A^{b}(x)\right)^{\prime} d x=A^{b}(+\infty)-A^{b}(t)=I_{G}-A^{b}(t)
$$

( $I_{G}$ is the identity operator in $G$ ), we obtain that

$$
\begin{gathered}
J_{1}=\iint_{\xi \leq t}\left(A^{b}(t) \Phi^{*}(t) g(t) \mid \Phi^{*}(\xi) g(\xi)\right)_{G} d t d \xi-\iint_{\xi \leq t}\left(\Phi^{*}(t) g(t) \mid \Phi^{*}(\xi) g(\xi)\right)_{G} d t d \xi= \\
=(U g \mid g)-\iint_{\xi \leq t}\left(\Phi^{*}(t) g(t) \mid \Phi^{*}(\xi) g(\xi)\right)_{G} d t d \xi ; \\
J_{2}=\iint_{t \leq \xi}\left(A^{b}(\xi) \Phi^{*}(t) g(t) \mid \Phi^{*}(\xi) g(\xi)\right)_{G} d t d \xi-\iint_{t \leq \xi}\left(\Phi^{*}(t) g(t) \mid \Phi^{*}(\xi) g(\xi)\right)_{G} d t d \xi= \\
=\left(U^{*} g \mid g\right)-\iint_{t \leq \xi}\left(\Phi^{*}(t) g(t) \mid \Phi^{*}(\xi) g(\xi)\right)_{G} d t d \xi .
\end{gathered}
$$

It thus follows that

$$
J=J_{1}+J_{2}=\left(g \mid U^{*} g\right)+\left(U^{*} g \mid g\right)-\int_{\mathbb{R}} \int_{\mathbb{R}}\left(\Phi^{*}(t) g(t) \mid \Phi^{*}(\xi) g(\xi)\right)_{G} d t d \xi .
$$

Since

$$
\int_{\mathbb{R}} \int_{\mathbb{R}}\left(\Phi^{*}(t) g(t) \mid \Phi^{*}(\xi) g(\xi)\right)_{G} d t d \xi=\sum_{j=1}^{n}\left|\left(g \mid \varphi_{j}\right)\right|^{2}=\left\|P^{\perp} g\right\|^{2},
$$

we have $J=\left(g \mid U^{*} g\right)+\left(U^{*} g \mid g\right)-\left\|P^{\perp} g\right\|^{2}$. 
Proof of Theorem 1. It follows from Lemma 3 that for an arbitrary $g \in C_{0}$

$$
\begin{gathered}
\left\|V^{*} g\right\|^{2}=\left((I-U)^{*} g \mid(I-U)^{*} g\right)=\|g\|^{2}+\left\|U^{*} g\right\|^{2}-\left(U^{*} g \mid g\right)-\left(g \mid U^{*} g\right)= \\
=\|g\|^{2}-\left\|P^{\perp} g\right\|^{2}=\|P g\|^{2}
\end{gathered}
$$

i.e., $\left(V V^{*} g \mid g\right)=(P g \mid g)$. Therefore, we get the equality $V V^{*}=P$. In view of Corollary 1, the operator $V$ is isometric and upper-triangular. The theorem is proved.

Acknowledgements. The authors express their gratitude to Dr. Ya. V. Mykytyuk for the formulation of the problem and thank him for his ideas to solve it.

\section{REFERENCES}

1. I. Gohberg, M. Krein, Theory of Volterra operators in Hilbert space and its applications, Nauka Publ., Moscow, 1967 (in Russian); Engl. transl.: Amer. Math. Soc. Transl. Math. Monographs, V.24, Amer. Math. Soc., Providence, RI, 1970.

2. S. Albeverio, R. Hryniv, Ya. Mykytyuk, Factorisation of non-negative Fredholm operators and inverse spectral problems for Bessel operators, Integr. equ. oper. theory, 64 (2009), 301-323.

3. D.R. Larson, Nest algebras and similarity transformations, Ann of Math. (2), 121 (1985), №2, 409-427.

Ivan Franko National University of Lviv

Lviv, Ukraine

n.sushchyk@gmail.com

Peeklogic

Lviv, Ukraine

dehnerysvitalii@gmail.com 